\title{
A U-HPLC-ESI-MS/MS-Based Stable Isotope Dilution Method for the Detection and Quantitation of Methotrexate in Plasma
}

\author{
Ethan den Boer, MSc, * Sandra G. Heil, PhD, * Bertrand D. van Zelst, BaSc, * \\ Roland J.W. Meesters, PhD, † Birgit C.P. Koch, PharmD, PhD, $\neq$ Mariël L. te Winkel, MD,, \\ Marry M. van den Heuvel-Eibrink, MD, PhD, $\S$ Theo M. Luider, PhD, $\dagger$ and Robert de Jonge, PhD*
}

\begin{abstract}
Introduction: High-dose methotrexate (MTX) is used in the treatment of proliferative diseases such as acute lymphoblastic leukemia. Therapeutic drug monitoring of plasma MTX is important to monitor efficacy and adverse events. The authors aimed to develop a liquid chromatography, electrospray ionization, tandem mass spectrometry (LC-ESI-MS/MS)-based method to determine MTX in plasma for therapeutic drug monitoring and pharmacokinetic studies.
\end{abstract}

Methods: Samples were analyzed using a Waters Acquity UPLC and Quattro Premier XE. A Waters Acquity UPLC BEH C18 column $(2.1 \mathrm{~mm} \times 100 \mathrm{~mm}, 1.7 \mu \mathrm{m})$ was used running an isocratic mobile phase of $21 \%$ methanol and $10 \mathrm{mM}$ ammonium bicarbonate. The electrospray was operated in the positive ionization mode monitoring the following mass transitions: $\mathrm{m} / z$ 455.2 $>308.2$ for MTX and $\mathrm{m} / \mathrm{z}$ $458.2>311.2$ for MTXd3. The analysis combined straightforward sample preparation, consisting of dilution and protein precipitation, with a 3-minute run time.

Results: The method was linear up to $50 \mu \mathrm{M}\left(r^{2}>0.99\right)$, and the coefficient of variation was $<6 \%$ for intraday and $<10 \%$ for interday precision. Average recovery was $99 \%$. There were no significant matrix effects. The lower limit of quantitation, defined as the lowest concentration at which the coefficient of variation $<20 \%$ and $S / N>$ 1:10, was $5 \mathrm{nM}$. Method comparison with the Abbott TDx fluorescent polarization immunoassay (FPIA) showed excellent agreement, and a small but significant negative constant bias was detected (LC$\mathrm{MS} / \mathrm{MS}=0.98 \times$ FPIA -7.3$)$.

Conlusions: The authors developed a specific and sensitive stable isotope dilution LC-ESI-MS/MS method to monitor MTX concen-

Received for publication February 17, 2012; accepted April 23, 2012.

From the *Department of Clinical Chemistry; †Department of Neurology; $\ddagger$ Hospital Pharmacy; and §Department of Oncology/Hematology, Erasmus MC, Sophia Children's Hospital, Erasmus MC, University Medical Centre Rotterdam, 's Gravendijkwal, Rotterdam, the Netherlands.

Supported by the Dutch Arthritis Association (nr. 06-02-402, 09-1-402), Stichting Kinderen Kankervrij (KiKa, nr. 67), and an Erasmus MC translational grant.

Supplemental digital content is available for this article. Direct URL citations appear in the printed text and are provided in the HTML and PDF versions this article on the journal's Web site (www.drug-monitoring.com).

The authors declare no conflicts of interest.

Correspondence: Robert de Jonge, PhD, Department of Clinical Chemistry, Erasmus MC, 's Gravendijkwal 230, room L-137, 3015 CE Rotterdam, the Netherlands (e-mail: r.dejonge@erasmusmc.nl)

Copyright (C) 2012 by Lippincott Williams \& Wilkins trations in plasma within the clinically relevant range. The method can be easily applied in clinical laboratories because it combines straightforward sample pretreatment with LC-MS/MS.

Key Words: methotrexate, human plasma, mass spectrometry, stable isotope, FPIA, therapeutic drug monitoring

(Ther Drug Monit 2012;34:432-439)

\section{INTRODUCTION}

Methotrexate (MTX) is a folate antagonist that has been used in clinical practice for 5 decades. It is used in the treatment of acute lymphoblastic leukemia (ALL), ${ }^{1,2}$ which is the most frequent cancer in children aged less than 15 years, ${ }^{3}$ and other proliferative diseases. ${ }^{4}$ Treatment is often limited due to severe toxicity, ${ }^{5}$ and monitoring of plasma MTX is important to assess efficacy and adverse events. ${ }^{3,6,7}$

Plasma MTX is commonly measured using immunoassays such as the fluorescent polarization immunoassay (FPIA) and enzyme-multiplied immunoassay technique ${ }^{8}$ from Abbott and Siemens, respectively. Immunoassays need limited sample preparation, and relatively fast turnaround times can be achieved, which make them suitable for routine clinical application.

However, immunoassays are expensive and are hampered by interference such as cross-reaction with folates and MTX metabolites, leading to low specificity. ${ }^{9-12}$ Although immunoassays are easy to implement and run, the large plasma concentration range of MTX in high-dose (HD) MTX treated patients (20 $\mathrm{nM}$ to $100 \mu \mathrm{M}$ ) imposes the need for testing samples in serial dilution. ${ }^{12,13}$ This compromises the assay's throughput unless an extensive infrastructure is present. Furthermore, immunoassays generally display poor sensitivity ${ }^{9}$; the lower limit of quantitation (LLOQ) of most immunoassays for MTX lies in the $20-\mathrm{nM}$ range. Although this is below the clinically relevant concentration [therapeutic drug monitoring (TDM) of MTX plasma concentrations generally stops $<100 \mathrm{nM}$, pharmacokinetic studies might benefit from a lower LLOQ.

Several high-performance liquid chromatography (HPLC)-based methods have been developed to improve specificity and sensitivity of plasma MTX detection. Commonly used detection methods include fluorescence detection, ${ }^{6,14-16}$ UV detection, ${ }^{17}$ and mass spectrometry. ${ }^{18-21}$ Although HPLCbased methods have many advantages, most HPLC methods rely on UV detection or fluorescence, which makes these 
methods subject to interference by other compounds, most notoriously folates and the deconjugated MTX form 4-amino-4deoxy-N-methylpteroic acid (DAMPA), ${ }^{22}$ leading to poor specificity and lower sensitivity (high LLOQ).

Liquid chromatography-tandem mass spectrometry (LC-MS/MS) is becoming a more routine method in the clinical laboratory. ${ }^{23} \mathrm{LC}-\mathrm{MS} / \mathrm{MS}$ is generally more specific than either conventional HPLC or immunoassay methods. In addition, these LC-MS/MS-based methods will in most cases result in shorter analysis times than do other HPLC methods, leading to higher throughput. ${ }^{24}$ Therefore, we aimed to develop and validate a new LC-MS/MS method for the measurement of MTX in plasma.

\section{METHODS}

\section{Chemicals and Blood Specimens}

MTX was purchased from Schircks Laboratories (Jona, Switzerland). Deuterium-labeled MTX (MTXd3) was used as internal standard (IS) and was purchased from Toronto Research Chemicals Inc (North York, Canada). Chemical structures of MTX and the deuterated analog are illustrated in Figure 1. LC-MS grade methanol and water were obtained from Biosolve (Valkenswaard, The Netherlands). Ammonium bicarbonate and perchloric acid $(70 \% \mathrm{vol} / \mathrm{vol})$ were purchased from Sigma (Zwijndrecht, The Netherlands). Ammonia ( $25 \% \mathrm{vol} / \mathrm{vol}$ ) was purchased from Merck (Schiphol-Rijk, The Netherlands).
Blinded drug-free and patient blood samples were used for development and validation of the method. Blinded samples were left over from routine analysis at the department of Clinical Chemistry and the Hospital Pharmacy; hence, no medical-ethical approval was necessary for this study.

\section{Instruments}

Analyses were performed on an LC-MS/MS system consisting of a Waters Acquity UPLC (Waters Corporation, Etten-Leur, The Netherlands) equipped with a $20-\mu \mathrm{L}$ sample loop and a Quattro Premier XE (Waters Corporation, EttenLeur, The Netherlands) triple quadrupole mass spectrometer with an electrospray ionization source operated in the positive ionization mode.

\section{Chromatographic Conditions}

Chromatography was performed using partial loop injection of $10-\mu \mathrm{L}$ sample on a Waters Acquity UPLC BEH C18 column $(2.1 \mathrm{~mm} \times 100 \mathrm{~mm}, 1.7 \mu \mathrm{m})$ maintained at $35^{\circ} \mathrm{C}$. The mobile phase consisted of $10 \mathrm{mM}$ ammonium bicarbonate adjusted to $\mathrm{pH} 10$ with $25 \%$ ammonia as the aqueous component (A), and methanol as the organic component (B). The system was maintained at a flow rate of $0.3 \mathrm{~mL} / \mathrm{min}$. keeping an isocratic concentration of $21 \% \mathrm{~B}$. After every 80 samples, the column was flushed or 10 minutes with $100 \%$ methanol at a flow rate of $0.3 \mathrm{~mL} / \mathrm{min}$ followed by reequilibration with $21 \% \mathrm{~B}$ at a flow rate of $0.3 \mathrm{~mL} / \mathrm{min}$.

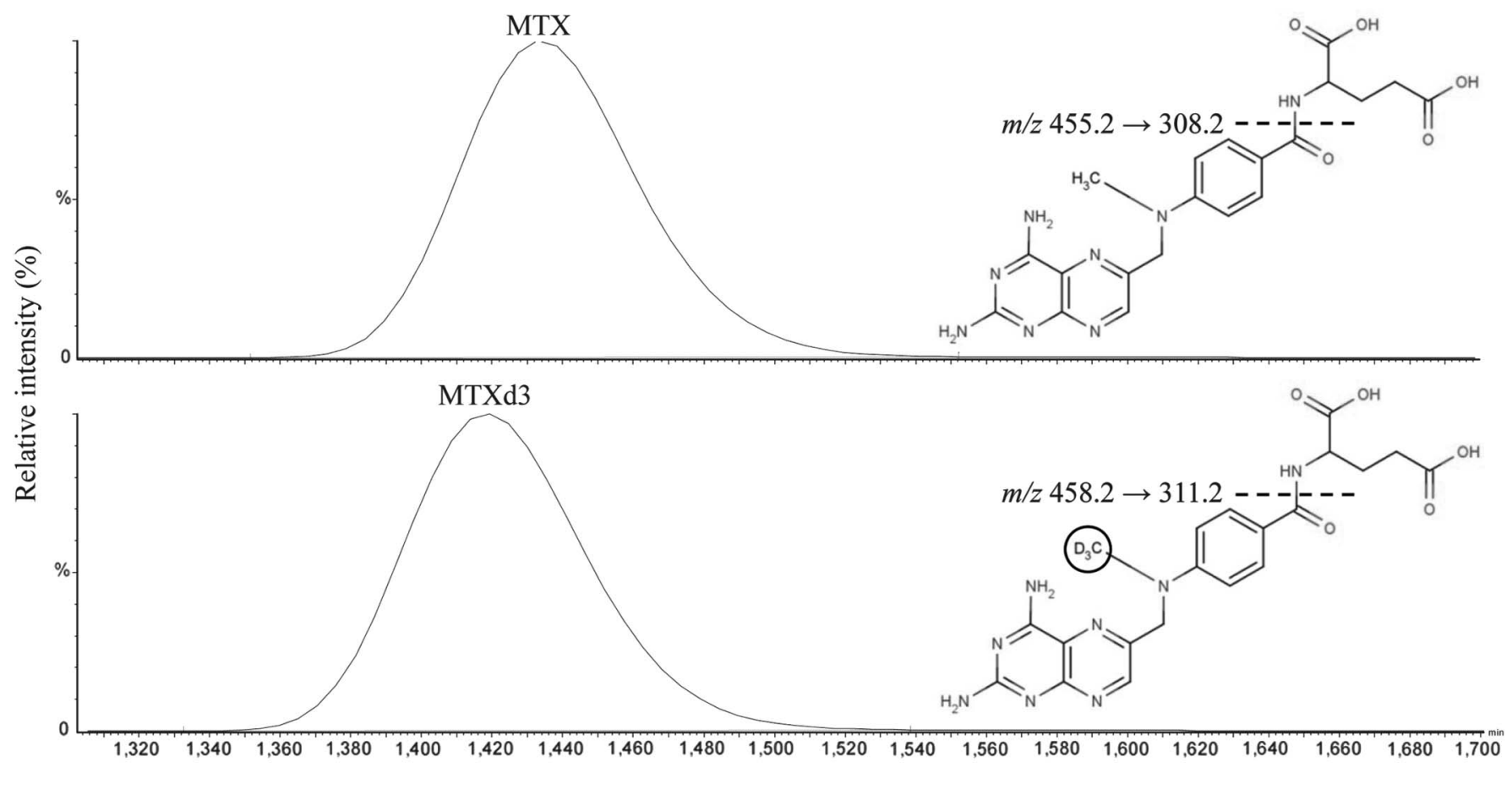

Time (min)

FIGURE 1. Chromatograms and chemical structures of MTX and IS (MTXd3). The location of the deuterium-enriched methylgroup is highlighted. The SRM for both MTX and MTXd3 are depicted in the table. 


\section{Mass-Spectrometery Detection}

The electrospray ionization source was operated in the positive mode with the following selected reaction monitoring (SRM) mass transitions: $m / z 455.2>308.2$ for MTX and $458.2>311.2$ for MTXd3 (Fig. 1).

Other mass-spectrometer settings were as follows: capillary voltage $1.00 \mathrm{kV}$, cone voltage $30 \mathrm{~V}$, collision energy $20 \mathrm{eV}$, source temperature $120^{\circ} \mathrm{C}$, desolvation temperature $350^{\circ} \mathrm{C}$ at a gas flow of $700 \mathrm{~L} / \mathrm{h}$, and cone gas flow $50 \mathrm{~L} / \mathrm{h}$. Argon was used as collision gas at a flow rate of $0.20 \mathrm{~mL} / \mathrm{min}$.

\section{Preparation of Standards}

Stock solutions were prepared in $0.1 \mathrm{M}$ ammonia at concentrations of $1836.6 \mu \mathrm{M}$ for MTX and $10 \mu \mathrm{M}$ for MTXd3. Both stock solutions were stored at $-80^{\circ} \mathrm{C}$. The stock solution of MTXd3 was diluted with LC-MS grade water to a working solution of $100 \mathrm{nM}$ to serve as the IS. The working solution was prepared freshly for each batch of prepared samples.

\section{Preparation of Calibrators and Quality Control Samples}

Frozen drug-free plasma was thawed at room temperature while rotating and then spiked with MTX to a final concentration of $50 \mu \mathrm{M}$. A 16-point calibration range was obtained by $1: 1$ serial dilution, containing $3.05 ; 6.10 ; 12.21$; $24.41 ; 48.83 ; 97.66 ; 195.31 ; 391.63 ; 781.25 ; 1562.5 ; 3125$; 6250; 12,500; 25,000; and 50,000 nM MTX and a blank sample $(0 \mathrm{nM})$. A fresh calibration curve was prepared for each run.

Quality control (QC) samples were prepared in drugfree plasma at 5 different concentrations: $10 ; 50 ; 500 ; 5000$; and 50,000 nM MTX. Aliquots were stored at $-80^{\circ} \mathrm{C}$ and thawed at $4^{\circ} \mathrm{C}$ while rotating.

\section{Sample Preparation}

Plasma was obtained by centrifuging $\mathrm{K}_{2}$-ethylenediaminetetraacetic acid (EDTA) whole-blood tubes at $2700 \mathrm{~g}$. A $50-\mu \mathrm{L}$ plasma aliquot was added to $450-\mu \mathrm{L}$ drug-free plasma and then supplemented with $500 \mu \mathrm{L}$ cold IS solution $(100 \mathrm{nM}$ MTXd3 in $\mathrm{H}_{2} \mathrm{O}$ ). Protein precipitation was done by the addition of $800 \mu \mathrm{L}$ of cold $16 \%$ perchloric acid to the diluted sample while vortexing. The samples were placed on ice for 30 minutes to enhance precipitation, after which they were centrifuged for 10 minutes at $2700 \mathrm{~g}$. The clear supernatant was transferred to a sample vial and placed in the autosampler of the LC-MS/MS system maintained at $4^{\circ} \mathrm{C}$. The injection volume was $10 \mu \mathrm{L}$.

Calibrators and QC samples were also prepared as described above. Concentrations were multiplied by 10 to correct for dilution.

\section{Validation Procedures}

Method validation was adapted from the 2001 Food and Drug Administration guidelines ${ }^{25}$ and Matuszewski et al. ${ }^{26}$ It consisted of selectivity, IS integrity, carryover, stability (short/long-term storage, freeze/thaw), recovery, matrix effects, linearity, LLOQ, precision (interday/intraday), and accuracy in comparison to FPIA (gold standard). All samples were measured in duplicate unless otherwise specified.

Selectivity of the detection was determined by measuring 20 different drug-free samples, which were prepared as described in the "Materials and Methods" section but replacing MTX and IS solution with LC-MS grade water. The average background signal was calculated for both MTX and MTXd3 and was used as noise when calculating the signal/noise ratio.

IS integrity was tested by measuring the presence of MTX, MTX mass +1 , MTX mass +2 , and MTX mass +3 in LC-MS/MS grade water spiked with $100 \mathrm{nM}$ IS.

Carryover was assessed by triplicate measurement of a spiked plasma sample with high concentration $(50 \mu \mathrm{M})$ directly followed by triplicate measurement of a sample with low $(50 \mathrm{nM})$ concentration and calculated as $\left[\left(\mathrm{Low}_{1}-\mathrm{Low}_{3}\right) /\right.$ $\left.\left(\mathrm{High}_{3}-\mathrm{Low}_{3}\right)\right] \times 100 \%{ }^{27}$ This was repeated in 3 different plasma batches.

Stability of MTX in plasma was studied by measuring the stored QC samples after 1 week, 6 weeks, and 6 months at $-80^{\circ} \mathrm{C}$. The stability of MTX after sample preparation was studied by storing a prepared concentration range (3-50,000 $\mathrm{nM}$ ) in the autosampler at $4^{\circ} \mathrm{C}$ for 16 days. Freeze/thaw stability was investigated by measuring QC samples after 3 successive cycles of freezing at $-80^{\circ} \mathrm{C}$ and unassisted thawing on ice. All the samples were measured 5 times from the same vial, and the results from these stability experiments were compared with those of freshly prepared and directly measured samples.

Recovery of the sample preparation was determined by spiking 10 different blank plasma samples with 3 different MTX concentrations $(50,500,5000 \mathrm{nM})$ and IS (100 nM) before and after sample preparation. The recovery was calculated with the following formula: Recovery $(\%)=($ MTX spiked before sample prep/MTX spiked after sample prep) $\times 100 \%$.

Matrix effects can have a detrimental effect on MSbased analysis, ${ }^{28}$ and both absolute and relative matrix effects can occur. ${ }^{26}$ Absolute matrix effect was defined as the difference in response between analyte spiked in sample matrix versus analyte spiked in pure solvent. A relative matrix effect is described as the variation in response between different samples in a similar matrix and can be expressed as the coefficient of variation (CV) between the slopes of reference curves made in different samples of the same matrix. The evaluation of matrix effects was performed according to the recommendations of Matuszewski et al. ${ }^{26}$ Ten reference curves were prepared by spiking 10 different drug-free plasma batches with 5 concentrations $(10 \mathrm{nM}, 50 \mathrm{nM}$, $500 \mathrm{nM}, 5 \mu \mathrm{M}$, and $50 \mu \mathrm{M})$ of MTX after sample preparation. The slopes of these reference curves in the different drug-free plasma batches were compared with the slope of a reference curve prepared in purified water to determine the absolute matrix effect. The absolute matrix effect was defined as: Absolute matrix effect $=($ mean slope plasma sample/slope distilled water) $\times 100 \%$. By comparing the reference curves of the 10 standard lines from the 10 different plasma samples the relative matrix effect was calculated, which was expressed as: Relative matrix effect $=100 \%-\mathrm{CV} \%$ of the slopes of the 10 reference curves. Furthermore, the maximum slope 
difference was calculated as the difference in percentage between the lowest slope and highest slope of the 10 reference curves. All matrix effects were calculated with and without correction by the IS. Slopes were calculated by linear regression analysis.

Linearity of the method was determined by spiking drugfree plasma with 22 different concentrations of MTX in a range of 3-250 $\mu \mathrm{M}$. This range reflects the full clinical range of MTX concentrations reported the past year at the Hospital Pharmacy of the Erasmus MC. The samples were measured 5 times from the same vial, and the experiment was repeated in a second drug-free plasma batch. Linearity was considered acceptable if the coefficient of determination $\left(R^{2}\right)$ was $>0.99$ for each calibration curve and if the lack of fit was $<3.75$.

$L L O Q$ was determined by spiking drug-free plasma samples with different levels of MTX covering a range of 3-50,000 $\mathrm{nM}$. These were measured 10 times from the same vial for each concentration level and the experiment was repeated in a second drug-free plasma batch. The LLOQ was defined as the lowest concentration of a serial dilution with a $\mathrm{CV} \%<20 \%$, while having an area of at least 10 times the average area of the drug-free plasma samples used for the selectivity experiment and 5 times the area of the blank sample $(0 \mathrm{nM})$.

Precision and accuracy were determined by measuring QC samples 5 times on 20 consecutive working days for interday precision. The QC samples were thawed and prepared as described in the "Materials and Methods" section. Everyday, a new batch of drug-free plasma was used for the dilution of the QC samples. The intraday precision was assessed by measuring QC samples 20 times in 1 run. This was repeated on a different day with another drug-free plasma batch for the dilution of the QC samples. Precision was expressed as $\mathrm{CV} \%$ and accuracy as bias (\%error).

\section{Method Comparison}

Blood samples from ALL patients were collected in $\mathrm{K}_{2}$-EDTA tubes from November 2009 to March 2011 by the Hospital Pharmacy of the Erasmus MC. These were used for routine measurement of plasma MTX using the FPIA on an Abbott TDx FLx Immunology Analyzer (Abbott Diagnostics, Hoofddorp, The Netherlands).

In total, 194 plasma samples from 34 ALL patients receiving $2-5 \mathrm{~g} / \mathrm{m}^{2}$ MTX for 24 hours were measured using both FPIA and LC-MS/MS. After measurement by the Hospital Pharmacy, surplus plasma was kept at $-20^{\circ} \mathrm{C}$ before measurement by LC-MS/MS. All the samples were measured twice from the same vial on the LC-MS/MS to be able to detect carryover.

The samples were measured in random order to reduce sample selection bias. Samples were remeasured by LC-MS/ MS when (1) duplicate measurement differed $>15 \%$, (2) samples were below the LLOQ, or (3) above the upper limit of quantitation. All the samples that were remeasured were both obtained from the original prepared sample and from freshly prepared sample. Samples that were below the LLOQ were repeated undiluted, whereas samples that were above the upper limit of quantitation were diluted 1:100 in drug-free plasma before preparation.

\section{Statistics}

Quantitation was performed using peak area ratio of analyte to IS. Microsoft Excel and Analyse-it for Microsoft Excel (version 2.20) were used to perform linear regression (matrix effects studies, calibration lines, linearity). Validation studies were performed according to CLSI EP-6 and Passing and Bablok regression analysis with $95 \%$ confidence intervals (method comparison). Precision was expressed as coefficients of variation $(\mathrm{CVs}): \mathrm{CV} \%=(\mathrm{SD} / \mathrm{mean}) \times 100 \%$. MassLynx software (Waters) was used for the analysis of chromatograms. The processing of MS data was done using QuanLynx software (Waters).

\section{RESULTS}

\section{Method Performance}

To establish the appropriate MS conditions, a standard solution was infused directly into the MS for optimization. Collision-induced dissociation of the protonated molecules was performed, and the product ions with the highest abundance were selected for SRM analysis. Mass transitions obtained in positive mode were $455.2>308.2$ for MTX and $458.2>311.2$ for MTXd3 (Fig. 1). Other mass transitions were detected but their $S / N$ ratio was too low to be used for quantitation.

When measuring 20 drug-free samples, the signal did not rise above the noise, showing good selectivity of the assay and that no interferences were detected. $100 \mathrm{nM}$ MTXd3 in water yielded $0 \%, 0 \%, 3 \%$, and $97 \%$ for MTX, MTX mass + 1 , MTX mass + 2, and MTX mass +3 , respectively, showing good integrity and purity. Mean carryover was $0.021 \%$.

The results for the stability experiments are presented in Table 1. Untreated plasma samples were stable when stored for 1 week at $-80^{\circ} \mathrm{C}$ (mean recovery: $106 \%$ ), 6 weeks at $-80^{\circ} \mathrm{C}$ (mean recovery: $106 \%$ ), or 6 months at $-80^{\circ} \mathrm{C}$ (mean recovery: 107\%). After preparation, the samples were stable when kept in the autosampler at $4^{\circ} \mathrm{C}$ for up to 16 days (mean recovery: 102\%). Furthermore, this was confirmed in 11 patient samples that were remeasured after 1 week at $4^{\circ} \mathrm{C}$ (mean recovery: 110\%). Three subsequent freeze-thaw cycles did not significantly influence MTX concentration. The observed decrease in response after freeze-thaw was 3\%.

Average recovery ranged from $96 \%$ to $102 \%$ for all concentrations $(50,500,5000 \mathrm{nM})$ of MTX with a CV\% of $<15 \%$ for each concentration when based on the area of the MTX peaks. After correction with the IS, average recovery ranged from $98 \%$ to $101 \%$ for all concentrations with a $\mathrm{CV} \%$ of $<6 \%$ for each concentration (Table 2 ).

TABLE 1. Recovery of MTX in Plasma

\begin{tabular}{lccc}
\hline $\begin{array}{c}\text { Sample Concentration } \\
(\mathbf{n M})(\mathbf{n}=\mathbf{1 0})\end{array}$ & $\begin{array}{c}\text { Measured } \\
\text { Concentration } \\
(\mathbf{n M} \pm \mathbf{S D})\end{array}$ & $\begin{array}{c}\text { Average } \\
\text { Recovery } \\
\mathbf{( \% )} \pm \mathbf{S D})\end{array}$ & $\mathbf{C V \%}$ \\
\hline 50 & $50.4 \pm 2.5$ & $101 \pm 5$ & 5 \\
500 & $503.2 \pm 14.6$ & $99 \pm 5$ & 5 \\
5000 & $5001.6 \pm 175.7$ & $98 \pm 6$ & 6 \\
\hline
\end{tabular}


TABLE 2. Stability of Stored Spiked Plasma

\begin{tabular}{llc}
\hline \multicolumn{1}{c}{ Conditions } & \multicolumn{1}{c}{$\begin{array}{c}\text { Average } \\
\text { Recovery (\%) } \\
\text { (Range) }\end{array}$} \\
\hline Before sample preparation* & $1 \mathrm{wk}-80^{\circ} \mathrm{C}$ & $106(95-115)$ \\
& $6 \mathrm{wks}-80^{\circ} \mathrm{C}$ & $106(100-115)$ \\
& $6 \mathrm{mos}-80^{\circ} \mathrm{C}$ & $107(103-114)$ \\
Freeze/thaw* & $\left(3 \mathrm{Cycles}-80^{\circ} \mathrm{C}\right)$ & $97(92-99)$ \\
After sample preparation $\dagger$ & $1 \mathrm{~d} 4^{\circ} \mathrm{C}$ & $102(93-115)$ \\
& $1 \mathrm{wk} 4^{\circ} \mathrm{C}$ & $102(91-110)$ \\
& $2 \mathrm{wks} 4^{\circ} \mathrm{C}$ & $102(93-113)$ \\
\hline
\end{tabular}

*The average recovery represents the recovery of the different QC sample concentrations, measured in 5-fold and compared with the results of the freshly prepared QC samples.

$\dagger$ The average recovery represents the recovery of a prepared calibration curve, measured in 2-fold and compared with the results of the freshly prepared calibration curve.

The absolute matrix effect calculated without correction for the IS was $71 \%$ with a relative matrix effect of $81 \%$ and a maximum slope difference of $67 \%$, showing large variation between the different samples. After correction with the IS, the absolute matrix effect improved to $94 \%$ with a relative matrix effect of $98 \%$ and a maximum slope difference of $7 \%$. All slopes showed an $r^{2}>0.999$. Matrix effects tested in samples without dilution in drug-free plasma during sample preparation yielded similar results (Table 3 ).

The method was linear up to $50 \mu \mathrm{M}(y=-0.326+$ $0.997 x$ ) and subsequent validation samples and QC samples were analyzed using calibration curves of $3 \mathrm{nM}$ to $50 \mu \mathrm{M}$ (see Data, Supplemental Digital Content 1, http://links.lww.com/TDM/A15). These were linear $\left(r^{2}>\right.$ 0.998 , lack of fit $<0.85)$ throughout the validation.

When determining the LLOQ, the CV\% did not become $>20 \%$ at any concentration other than the blank (0 nM) sample (see Data, Supplemental Digital Content 2, http://links.lww.com/TDM/A16). In all samples, the signal to noise ratio was better than 10:1. The LLOQ was set at $5 \mathrm{nM}$ because at this concentration there was always (1) a visual and distinguishable peak present in every calibration curve and (2) a response 5 times higher than the response of the lowest sample of the calibration curve $(0 \mathrm{nM})$. The precision profile of the method is shown in Table 4. Precision ranged between $2.1 \%$ and $6.5 \%$ for intraday variation and between $5.8 \%$ and $11.0 \%$ for interday variation for the total range of tested MTX concentrations. The mean bias (\%error) ranged from $-11.3 \%$ to $14.5 \%$ for intraday precision and $-9.7 \%$ to $14.4 \%$ for the total range of tested MTX concentrations.

\section{Method Comparison}

The 194 HD-MTX patient plasma samples were compared between methods. Duplicate measurements on the LCMS/MS always showed a CV $<10 \%$ with the exception of 14 samples, of which 10 samples were below the LLOQ of either the FPIA or the LC-MS/MS and were therefore excluded from the comparison. The other 4 samples contained low concentrations of MTX (20-36 nM) and were preceded by high concentration samples (35,088-146,851 nM). The observed difference between duplicates $(6-45 \mathrm{nM})$ was similar to the expected carryover (7-31 nM) assuming the $0.02 \%$ found previously, suggesting carryover as the cause. Another 9 samples were excluded because MTX plasma concentrations were below the LLOQ of the FPIA and therefore not reported as numerical data but as " $<20 \mathrm{nM}$ " by FPIA. All the samples for which the $\mathrm{CV}$ was $>10 \%$ were remeasured both from the original prepared sample and from freshly prepared samples after which all samples had duplicates with a $\mathrm{CV}<10 \%$.

The comparison of the remaining 175 samples showed good agreement between methods: [LC-MS/MS $=0.98(95 \%$ CI: $0.95-1.02) \times$ FPIA $-7.3 \mathrm{nM}(95 \% \mathrm{CI}:-10.96$ to -5.34$)]$ (Fig. 2, insert). A slight, nonsignificant, proportional bias was present, and a small but significant constant bias of $-7.3 \mathrm{nM}$ was found for the complete sample set. Changing the method comparison to the low (0-500 nM), high (500-50,000 nM), or complete sample concentration range did not give a significant difference in slope or bias. For the lower concentration range $(0-500 \mathrm{nM})$, which contained $75 \%$ of all patient samples, good agreement was obtained [LC-MS/MS $=1.00$ (95\% CI: $0.94-1.06) \times$ FPIA -8.51 nM $(95 \%$ CI: -11.06 to -5.88$)$ ] (Fig. 2).

\section{DISCUSSION}

We developed a straightforward and robust LC-MS/ MS-based method for the measurement of MTX in human

TABLE 3. Matrix Effects of 10 Different Plasma Samples*

\begin{tabular}{|c|c|c|c|c|c|}
\hline Sample Concentration & & $\begin{array}{c}\text { Average Matrix } \\
\text { Effect }(\%)\end{array}$ & CV\% & $\begin{array}{c}\text { Relative Matrix } \\
\text { Effect }(\%)\end{array}$ & $\begin{array}{l}\text { Maximum Slope } \\
\text { Difference } \dagger(\%)\end{array}$ \\
\hline \multirow[t]{2}{*}{ Diluted plasma } & With IS & 94 & 2 & 98 & 7 \\
\hline & Without IS & 71 & 19 & 81 & 67 \\
\hline & Without IS & 72 & 17 & 93 & 67 \\
\hline
\end{tabular}

All slopes were $r^{2}>0.999$ when corrected for IS

*Matrix effect is expressed as percent recovery and calculated by comparing the slopes of reference curves made in 10 different plasma matrices with the slope of a reference curves made in water.

$\dagger$ The maximum slope difference shows the percent difference between the largest and smallest slope, indicating the variance between slopes in different matrices.

$\$ 1: 10$ in drug-free plasma 
TABLE 4. Intraday and Interday Precision

\begin{tabular}{|c|c|c|c|c|c|c|}
\hline \multirow[b]{2}{*}{ Expected Concentration (nM) } & \multicolumn{3}{|c|}{$\begin{array}{l}\text { Measured Concentration Intraday } \\
\text { Precision }(\mathrm{nM} ; \mathrm{n}=\mathbf{2 0})^{*}\end{array}$} & \multicolumn{3}{|c|}{$\begin{array}{l}\text { Measured Concentration Interday } \\
\text { Precision }(\mathbf{n M} ; \mathbf{n}=\mathbf{2 0}) \dagger\end{array}$} \\
\hline & Mean \pm SD & $\mathrm{CV} \%$ & Bias (\%error) & Mean \pm SD & $\mathrm{CV} \%$ & Bias (\%error) \\
\hline 10 & $11.4 \pm 0.76$ & 6.5 & 14.5 & $11.6 \pm 1.27$ & 11.0 & 14.4 \\
\hline 500 & $543.9 \pm 17.84$ & 3.3 & 8.8 & $536.3 \pm 40.43$ & 7.5 & 7.1 \\
\hline 5000 & $4947.4 \pm 168.85$ & 3.4 & -1.1 & $5073.1 \pm 465.15$ & 9.2 & 1.3 \\
\hline
\end{tabular}

*The intraday precision summarizes 20 replicate measurements at each concentration of MTX during 1 day.

$\dagger$ The interday precision summarizes 20 different experiments from 20 consecutive days with measurement done in 5 -fold each day.

EDTA plasma. Although other mass spectrometry-based methods have been reported for the detection of MTX in plasma, most methods do not use stable isotope-labeled IS. Furthermore, these methods generally have higher LLOQs ${ }^{29-31}$ or use more expensive and work-intensive sample preparation and machinery. ${ }^{18,32}$ When compared with traditional methods of MTX TDM (such as FPIA), our method has improved LLOQ and has a large dynamic range.

The presented method does not require expensive and time-consuming sample preparation, such as solid-phase extraction, and because of the use of a stable isotope-labeled IS, it is a very useful method for implementation within a clinical laboratory. The short analysis time of 3 minutes makes the method attractive for high-throughput settings and clinical studies. Improvements in LLOQ can be made with the addition of better sample cleanup, such as SPE, but this would also drive up the cost and preparation time of the method. Alternatively, LLOQ may be improved by reducing the dilution factor. However, due to the dilution of patient sample in drug-free plasma, less volume is needed making the method suitable for use in very small sample volumes such as in pediatric samples.

Although carryover is only $0.02 \%$, the difference in maximum plasma concentrations in HD-MTX treatment can be as high as 100 -fold. When measuring very low concentration samples $(<100 \mathrm{nM})$ after very high concentration samples $(>50 \mu \mathrm{M})$, this could lead to falsely increased MTX concentrations in the low concentration range as was seen when patient samples were measured in random order. Samples with a low concentration of MTX measured directly after samples with a high concentration of MTX had a discrepancy between duplicates that fitted roughly with the $0.02 \%$ carryover that was determined. The use of duplicate measurement is important to detect and counter this. For most TDM purposes, this will not pose any problems as the clinical cut-off for TDM is usually approximately $200 \mathrm{nM}$. However, when dealing with samples in the setting of carboxylase- $\mathrm{G} 2$ treatment, carryover might present a problem due to the large difference in MTX concentration between patient samples as a result of MTX breakdown by the enzyme. ${ }^{12,13,33}$
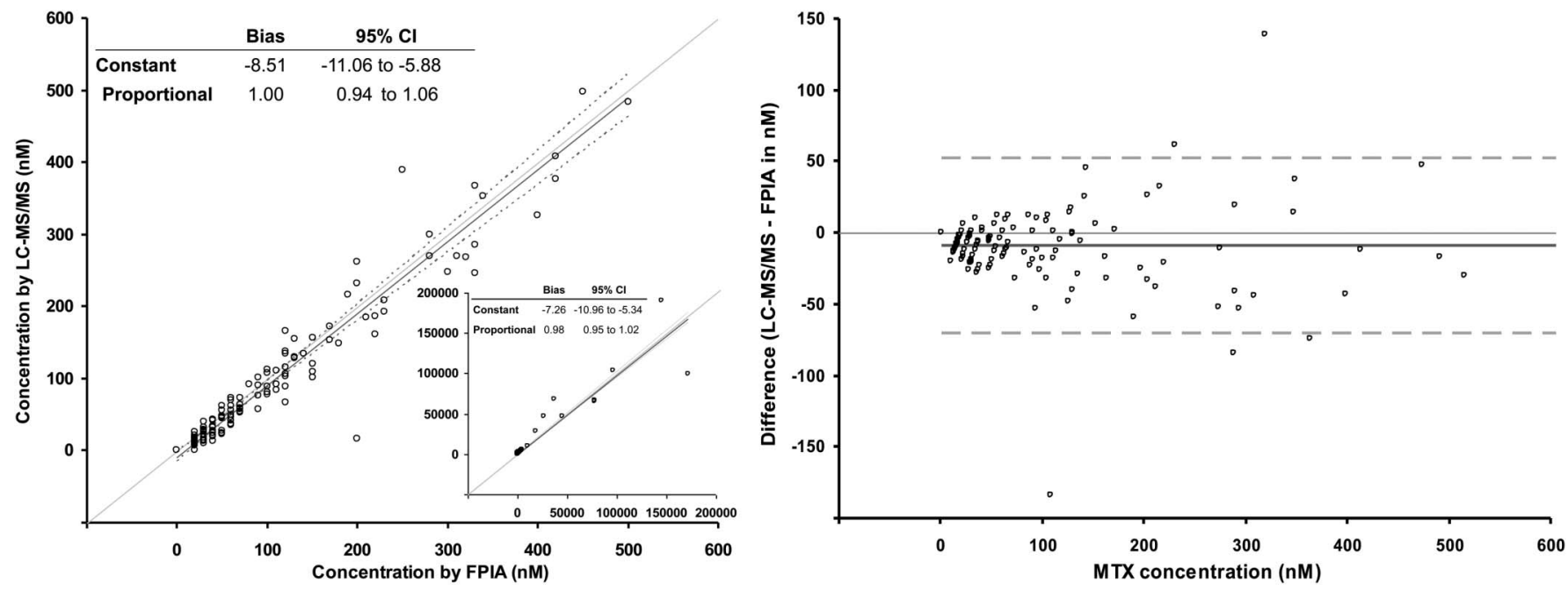

FIGURE 2. Comparison of plasma MTX between the Abbott FPIA on the TDx analyzer ( $x$-axis) compared with the LC-MS/MS method ( $y$-axis) $(n=175)$. Samples shown are in the range of $0-500 n M M T X$, comprising $75 \%$ of all patient samples $(n=131)$. Left panel: Passing and Bablok regression was used to compare both methods. The insert shows the comparison of the whole concentration range (0-170 $\mathrm{MM}$ MTX). Right panel: Bland-Altman plot of differences between observed MTX concentrations by FPIA and LC-MS/MS methods. Solid line represents bias $(-9.02 \mathrm{nM})$, and dotted lines represent $95 \%$ limits of agreement $(-70.13$ to 52.08$)$. 
Recovery based on peak area was moderate with high variance, but this was corrected for by the use of the stable isotope-labeled IS, leading to more reproducible measurements and a recovery of approximately $100 \%$. The use of a deuterium-labeled IS also compensated for matrix effects. When using the peak areas to calculate matrix effects, a prominent absolute matrix effect was detected, which was almost completely corrected for by the use of the IS. The relative matrix effect caused by using different plasma batches is also corrected for by use of the IS. Both the recovery and matrix effect experiments emphasize the need for a proper IS when working with complex matrices such as biological samples in an LC-MS/MS setting.

Matrix effects in undiluted samples were also very low after correction for the IS, which indicates that the dilution step in drug-free plasma is optional and leads to the possibility of measuring very low sample concentrations $(<$ LLOQ) without dilution.

As shown by the method comparison, a good agreement was found with the results of FPIA analysis. This correlation was somewhat unexpected due to reports about the poor specificity of the FPIA method. ${ }^{9,11,33}$ The few outliers that were found and the observed small constant bias might be caused by several reasons. First, the FPIA might measure higher concentrations because of its nonspecific measurement of MTX metabolites such as DAMPA. Second, differences might exist in the calibration between methods (traceability chain). Third, calibration errors within the laboratory might also have caused the constant bias between FPIA and LC-MS/MS. Because there is neither a reference method nor standard reference material for MTX in plasma, it is difficult to speculate about the accuracy of the methods. However, in line with Food and Drug Administration guidelines, our LC-MS/MS method showed a small positive bias (\%error $<7.1$ for interday and $<8.8$ for intraday) in the clinically relevant concentration range based on the spiked samples and the intraday recovery (Table 4). In the higher concentration range, the bias was slightly negative (\%error -9.7 for interday and -11.3 for intraday). In certain situations, immunoassays such as the FPIA method may be hampered by interference of MTX metabolites. For example, our method was able to measure the rapid disappearance of plasma MTX in patients treated with carboxylase-G2 for adverse events, whereas the FPIA method was hampered by falsely high MTX concentrations due to interference from the methotrexate metabolite DAMPA (data not shown). This observation was confirmed by the literature. . $^{12,13}$

In conclusion, we describe a LC-MS/MS-based method to measure MTX in plasma with minimal sample pretreatment, relative short analysis time, and good performance that can be applied to routine clinical TDM.

\section{REFERENCES}

1. Panetta J, Yanishevski Y, Pui CH, et al. A mathematical model of in vivo methotrexate accumulation in acute lymphoblastic leukemia. Cancer Chemother Pharmacol. 2002;50:419-428.

2. Rubino FM. Separation methods for methotrexate, its structural analogues and metabolites. J Chromatogr B Biomed Sci Appl. 2001;764: $217-254$.
3. Martelli N, Mathieu O, Margueritte G, et al. Methotrexate pharmacokinetics in childhood acute lymphoblastic leukaemia: a prognostic value? J Clin Pharm Ther. 2011;36:237-245.

4. Krajinovic M, Moghrabi A. Pharmacogenetics of methotrexate. Pharmacogenomics. 2004;5:819-834.

5. Faganel Kotnik B, Grabnar I, Bohanec Grabar P, et al. Association of genetic polymorphism in the folate metabolic pathway with methotrexate pharmacokinetics and toxicity in childhood acute lymphoblastic leukaemia and malignant lymphoma. Eur J Clin Pharmacol. 2011;67: 993-1006.

6. Uchiyama M, Matsumoto T, Jimi S, et al. Simple and sensitive HPLC method for the fluorometric determination of methotrexate and its major metabolites in human plasma by post-column photochemical reaction. Biomed Chromatogr. 2011;26:76-80.

7. Alnaim L. Therapeutic drug monitoring of cancer chemotherapy. J Oncol Pharm Pract. 2007;13:207-221.

8. Mendu DR, Chou PP, Soldin SJ. An improved application for the enzyme multipled immunoassay technique for caffeine, amikacin, and methotrexate assays on the Dade-Behring Dimension RxL Max clinical chemistry system. Ther Drug Monit. 2007;29:632-637.

9. Eksborg S, Albertioni F, Rask C, et al. Methotrexate plasma pharmacokinetics: importance of assay method. Cancer Lett. 1996;108:163-169.

10. Hayashi H, Fujimaki C, Tsuboi S, et al. Application of fluorescence polarization immunoassay for determination of methotrexate-polyglutamates in rheumatoid arthritis patients. Tohoku J Exp Med. 2008;215:95-101.

11. Albertioni F, Rask C, Eksborg S, et al. Evaluation of clinical assays for measuring high-dose methotrexate in plasma. Clin Chem. 1996;42:39-44.

12. Al-Turkmani MR, Law T, Narla A, et al. Difficulty measuring methotrexate in a patient with high-dose methotrexate-induced nephrotoxicity. Clin Chem. 2010;56:1792-1794.

13. Esteve MA, Devictor-Pierre B, Galy G, et al. Severe acute toxicity associated with high-dose methotrexate (MTX) therapy: use of therapeutic drug monitoring and test-dose to guide carboxypeptidase G2 rescue and MTX continuation. Eur J Clin Pharmacol. 2007;63:39-42.

14. Dervieux T, Orentas Lein D, Marcelletti J, et al. HPLC determination of erythrocyte methotrexate polyglutamates after low-dose methotrexate therapy in patients with rheumatoid arthritis. Clin Chem. 2003;49: $1632-1641$.

15. Hroch M, Tukova J, Dolezalova P, et al. An improved high-performance liquid chromatography method for quantification of methotrexate polyglutamates in red blood cells of children with juvenile idiopathic arthritis. Biopharm Drug Dispos. 2009;30:138-148.

16. McCrudden EA, Tett SE. Improved high-performance liquid chromatography determination of methotrexate and its major metabolite in plasma using a poly(styrene-divinylbenzene) column. J Chromatogr B Biomed Sci Appl. 1999;721:87-92.

17. Belz S, Frickel C, Wolfrom C, et al. High-performance liquid chromatographic determination of methotrexate, 7-hydroxymethotrexate, 5 -methyltetrahydrofolic acid and folinic acid in serum and cerebrospinal fluid. J Chromatogr B Biomed Appl. 1994;661:109-118.

18. Meesters RJ, den Boer E, Mathot RA, et al. Ultrafast selective quantification of methotrexate in human plasma by high-throughput MALDIisotope dilution mass spectrometry. Bioanalysis. 2011;3:1369-1378.

19. Barbieri A, Sabatini L, Indiveri P, et al. Simultaneous determination of low levels of methotrexate and cyclophosphamide in human urine by micro liquid chromatography/electrospray ionization tandem mass spectrometry. Rapid Commun Mass Spectrom. 2006;20:1889-1893.

20. Przybylski M, Preiss J, Dennebaum R, et al. Identification and quantitation of methotrexate and methotrexate metabolites in clinical high-dose therapy by high pressure liquid chromatography and field desorption mass spectrometry. Biomed Mass Spectrom. 1982;9:22-32.

21. Turci R, Fiorentino ML, Sottani C, et al. Determination of methotrexate in human urine at trace levels by solid phase extraction and high-performance liquid chromatography/tandem mass spectrometry. Rapid Commun Mass Spectrom. 2000;14:173-179.

22. van Haandel L, Becker ML, Leeder JS, et al. A novel high-performance liquid chromatography/mass spectrometry method for improved selective and sensitive measurement of methotrexate polyglutamation status in human red blood cells. Rapid Commun Mass Spectrom. 2009;23: 3693-3702.

23. Honour JW. Development and validation of a quantitative assay based on tandem mass spectrometry. Ann Clin Biochem. 2011;48:97-111. 
24. Shushan B. A review of clinical diagnostic applications of liquid chromatography-tandem mass spectrometry. Mass Spectrom Rev. 2010; 29:930-944.

25. CDER. Center for Drug Evaluation and Research (CDER) Guidance for Industry: Bioanalytical Method Validation. 2001. Available at: http:// www.fda.gov/cder/guidance/4252fnl.pdf.

26. Matuszewski BK, Constanzer ML, Chavez-Eng CM. Strategies for the assessment of matrix effect in quantitative bioanalytical methods based on HPLC-MS/MS. Anal Chem. 2003;75:3019-3030.

27. Broughton PM. Carry-over in automatic analysers. J Automat Chem. 1984;6:94-95.

28. Vogeser M, Seger C. Pitfalls associated with the use of liquid chromatography-tandem mass spectrometry in the clinical laboratory. Clin Chem. 2010;56:1234-1244.

29. Rule G, Chapple M, Henion J. A 384-well solid-phase extraction for LC/ MS/MS determination of methotrexate and its 7-hydroxy metabolite in human urine and plasma. Anal Chem. 2001;73:439-443.
30. Guo P, Wang X, Liu L, et al. Determination of methotrexate and its major metabolite 7-hydroxymethotrexate in mouse plasma and brain tissue by liquid chromatography-tandem mass spectrometry. J Pharm Biomed Anal. 2007;43:1789-1795.

31. Koufopantelis P, Georgakakou S, Kazanis M, et al. Direct injection liquid chromatography/positive ion electrospray ionization mass spectrometric quantification of methotrexate, folinic acid, folic acid and ondansetron in human serum. J Chromatogr B Analyt Technol Biomed Life Sci. 2009;877:3850-3856

32. Steinborner S, Henion J. Liquid-liquid extraction in the 96-well plate format with SRM LC/MS quantitative determination of methotrexate and its major metabolite in human plasma. Anal Chem. 1999;71: $2340-2345$

33. Kumar VS, Law T, Kellogg M. Liquid chromatography-tandem mass spectrometry (LC-MS-MS) method for monitoring methotrexate in the setting of carboxypeptidase-G2 therapy. Methods Mol Biol. 2010;603: 359-363. 